\title{
Risk Evaluation of Biomass Power Generation in Different Regions in China
}

\author{
Hong Fang and Xinglong Ren ${ }^{*}$ \\ College of Science, Huazhong Agricultural University, Wuhan, China
}

\begin{abstract}
People have payed more and more attention to biomass energy as the problem of energy is becoming serious. Biomass power generation is an important application of biomass energy. Because the basis and condition of biomass power generation are different in different regions, a reasonable risk evalution can help them to find their correct orientations. In this paper, ten indexes from five subsystems are chosen to do single subsystem risk evaluation, comprehensive evaluation and coordination degree evaluation respectively. According to the evaluating results, both the advantage and deficiency are pointed out in different regions in China.
\end{abstract}

Keywords-biomass energy; power generation; coordination degree; risk evaluation; entropy

\section{INTRODUCTION}

As the development of social economy, the energy problem is becoming a bottleneck of the economic development in all countries in the world[1]. The utilization of renewable energy are payed more and more attention by then. For its cleaning and safety, the biomass energy may be one of the important choice to be treated as the main source of human energy after the fossil energy.

Power generation of biomass energy is an important subject based on the strong demand of electric power in the market and the high efficiency utilization technology. In the process, forestry, agricultural and industrial waste, city life rubbish, waste water and garbage all can be used as raw material to generate electricity by pyrolysis, direct-fired or gasification[2].

In the United States, biomass power generation has became a mature industry, whose technology and management are advanced in the world, and the produced electric energy has acted as an irreplaceable role and been brought into the basic power network. In Denmark, almost all of the burning power projects are using biomass as the raw material. What is more, the coal-fired power plants are changing into biomass power generation plants in the country[3].

The first biomass power generation enterprise came into operation in China in 2004. Although more and more preferential policies were carried out to encourage biomass power generation, and there were more and more projects under construction, but the loss projects still accounted for a large part of the biomass power generation projects. The main reason is a lack of consideration of risk factors in development planning. In general, these risks mainly come from resource supply, technology, market, natural and policy environment[4].

\section{SELECTION OF RISK INDEXES}

Risk of raw materials is mainly embodied in the total amount $\left(\mathrm{x}_{1}\right)$ and stability $\left(\mathrm{x}_{2}\right)$ of supplied material. Biomass materials which Can be used for power generation mainly include crops straw, cattle feces, forestry biomass, waste water and garbage. Among them, the output of crops straw can be calculated according to the ratio of crops and straw, the output of cattle feces can be calculated according to the number of the cattle and the amount of feces of theirs in a unit cycle time, the output of forestry can be calculated by multiplying the total amount of trees and the coefficient of residual washed out, and the output of waste water and garbage can be directly obtained from some statistical yearbooks. We can get the standard total amount of biomass energy by multiplying the original amount of each kind of resource and a converting coefficient. In this paper, the stability of supplied material is expressed by the reciprocal of the deviation of the supplied amount of biomass energy in the past five years.

Risk of technology can be expressed by the developed level of science and technology $\left(\mathrm{x}_{3}\right)$ and technology investment funds $\left(\mathrm{x}_{4}\right)$. In this research, the total turnover in technology market in all regions can represent the developed level of science and technology, and the technology investment funds can be directly obtained from the statistical yearbook.

Risk of market can be expressed by the power consumption $\left(\mathrm{x}_{5}\right)$, the accepted degree of people $\left(\mathrm{x}_{6}\right)$ and the growth rate of power consumption $\left(\mathrm{x}_{7}\right)$. Among them, the power consumption can be directly obtained from the statistical yearbook, and the growth rate of power consumption can be expressed by the average growth rate of power consumption in the past five years. Because there is a direct relationship between the accepted degree of people to the biomass power generation and their education degree, the number of people who have at least a high school education will be used to express the accepted degree of people.

Risk of natural environment includes natural disasters losses $\left(\mathrm{x}_{8}\right)$ and investment to prevent and control the disasters $\left(\mathrm{x}_{9}\right)$, respectively expressed by direct economic losses and investment to prevent disasters.

Risk of policy environment basically depends on the policy support degree of biomass energy generation in each region $\left(\mathrm{x}_{10}\right)$, which can be calculated by the compensation degree of per unit power output obtained by biomass power generation enterprises. 


\section{DETERMINATION OF INDEX WEIGHT}

Considering the different dimensions of indexes, we can eliminate them as shown in the following formula:

$$
x_{i j}^{*}=\frac{x_{i j}-\min _{1 \leq j \leq n}\left\{x_{i j}\right\}}{\max _{1 \leq j \leq n}\left\{x_{i j}\right\}-\min _{1 \leq j \leq n}\left\{x_{i j}\right\}}
$$

where $x_{i j}$ is the value of index $i$ in region $j$.

The entropy method will be used to determine the weight of each index according to the following three steps.

Step 1:define the entropy of $m$ indexes in $n$ regions as the following formula:

$$
e_{j}=-k \sum_{i=1}^{m} p_{i j} \ln \left(p_{i j}\right)
$$

where

$$
\mathrm{p}_{\mathrm{ij}}=\frac{\mathrm{x}^{*}{ }_{\mathrm{ij}}}{\sum_{\mathrm{i}=1}^{\mathrm{n}} \mathrm{x}_{\mathrm{ij}}{ }^{*}}, \mathrm{k}=\frac{1}{\ln \mathrm{n}}
$$

Step 2:define the coefficient of variation of index $\mathrm{j}$ as the following formula:

$$
\mathrm{g}_{\mathrm{j}}=1-\mathrm{e}_{\mathrm{j}}
$$

Step 3:calculate the weight of index $\mathrm{j}$ as the following formula:

$$
w_{j}=\frac{g_{j}}{\sum_{j=1}^{m} g_{j}}
$$

Through consulting the China statistical yearbook, get and standardizing the original value of each index, then do according to the above entropy method and get the weight of each index as shown in table I.

TABLE I. WEIGHT OF EACH INDEX

\begin{tabular}{cc||cc}
\hline index & weight & index & weight \\
\hline $\mathrm{x}_{1}$ & 0.058 & $\mathrm{x}_{6}$ & 0.038 \\
$\mathrm{x}_{2}$ & 0.107 & $\mathrm{x}_{7}$ & 0.028 \\
$\mathrm{x}_{3}$ & 0.153 & $\mathrm{x}_{8}$ & 0.223 \\
$\mathrm{x}_{4}$ & 0.103 & $\mathrm{x}_{9}$ & 0.153 \\
$\mathrm{x}_{5}$ & 0.055 & $\mathrm{x}_{10}$ & 0.082 \\
\hline
\end{tabular}

From the table, we can see that the index of natural disasters losses $\left(\mathrm{x}_{8}\right)$ is the most important and the imdex of the growth rate of power consumption $\left(\mathrm{x}_{7}\right)$ has the weakest effect in all the indexes.

\section{RISK EVALUATION IN DIFFERENT REGIONS}

\section{A. Evaluation of Subsystem}

According to the weight, standardizing the value of each index in a subsystem and do weighted summation, then the evaluation value of each subsystem can be obtained.

The first five regions in supply risk subsystem are Shanghai, Beijing, Ningxia, Qinghai and Sichuan, whose evaluating value are $0.107,0.094,0.067,0.067$ and 0.061 respectivlely. And the last five regions after the group are Jiangsu, Shanxi, Anhui, Xinjiang and Shanxi. Shanghai and Beijing are first-tier cities in our country, there are a large number of day-life rubbish can be used as raw materials for biomass power generation so that the supply amount shows a kind of stability. Ningxia, Qinghai and Sichuan all have abundant natural resources, good farming and animal husbandry, which provides a good environment for the development of biomass power generation. Those low-ranking regions are neither important agricultural regions, nor have a rich forestry industry resources and a high proportion of population, and all these factors lead to a big risk of supply.

The first five regions in technical risk subsystem are Beijing, Jiangsu, Guangdong, Shanghai, Shandong. Shanghai, Guangdong and Beijing are core regions of culture and technology in our country, and there are many universities so that they have an incomparable advantages of science and technology. And Shandong, Jiangsu are the earliest supported regions in China to develop biomass power generation, so there is advanced technology in these regions. The last five regions are Xinjiang, Ningxia, Qinghai, Hainan and Xizang, and the main reason is their backward technology and lack of human resource, so these regions should be key supported regions.

The first five regions in natural risk subsystem are Sichuan, Gansu, Fujian, Jilin and Guangdong, and the last five regions are Shanghai, Xinjiang, Qinghai, Beijing and Tianjin. Xinjiang and Qinghai have a complex geological structure and a bad natural environment, and they are very vulnerable to the influence of natural resources so that there is a big risk in these regions. And Beijing, Tianjin, Shanghai have a poor environmental keeping ability, and their resisting ability of environmental damage is fragile so that they have a low rank too.

The first five regions in market risk subsystem are Beijing, Jiangsu, Shanghai, Guangdong and Shandong. They are densely populated regions, and there is a great demand of power consumption. Except Shandong, the other four regions are centers of many industries, so that their electricity demand is significantly higher than other regions'.

The first five regions in policy risk subsystem are Shandong, Neimonggu, Tianjin, Beijing and Xinjiang, and the 
last five regions are Gansu, Ningxia, Qinghai, Xizang and Shanxi.

\section{B. Comprehensive Evaluation}

The result of comprehensive evaluation of biomass power generation can be obtained according to the summation of evaluating values of all subsystems in each region. The larger the evaluating value is, the lower the risk is. Conversely, a smaller evaluating value shows a higher risk of biomass power generation in the region. The evaluating values of all regions are shown in table II.

TABLE II. VALUE OF COMPRHENSIVE EVALUATION

\begin{tabular}{cc||cc}
\hline region & $\begin{array}{c}\text { evaluating } \\
\text { value }\end{array}$ & region & $\begin{array}{c}\text { evaluating } \\
\text { value }\end{array}$ \\
\hline Beijing & 0.503 & Anhui & 0.148 \\
Tianjin & 0.396 & Heilongjiang & 0.145 \\
Sichuan & 0.354 & Chongqing & 0.143 \\
Guangdong & 0.330 & Jiangxi & 0.138 \\
Jiangsu & 0.290 & Jilin & 0.136 \\
Shandong & 0.266 & Gansu & 0.136 \\
Shanghai & 0.250 & Qinghai & 0.130 \\
Zhejiang & 0.227 & Xinjiang & 0.129 \\
Liaoning & 0.195 & Shanxi & 0.110 \\
Henan & 0.192 & Ningxia & 0.104 \\
Neimenggu & 0.192 & Shanxi & 0.078 \\
Hubei & 0.187 & Guangxi & 0.071 \\
Hunan & 0.171 & Xizang & 0.064 \\
Hebei & 0.170 & Guizhou & 0.059 \\
Fujian & 0.170 & Hainan & 0.057 \\
Yunnan & 0.166 & & \\
\hline
\end{tabular}

According to table II, the first five regions are Beijing, Tianjin, Guangdong, Jiangsu and Sichuan. Through analysis, we can see that Beijing, Guangdong and Tianjin are important technology centres in China, and there are a huge electricity demand and absolute advantage policies so that they have a low risk. And Xizang, Guangxi, Shanxi, Hainan although take a lead in the risk of raw materials, but their lack of policy and technology directly hinders their development of biomass power generation. The above problem is just our future direction.

\section{Evaluation of Coordination Degree}

The above subsystem evaluation and comprehensive evaluation only reflect the size of risk from a single aspect or a few aspects, but they can't reflect the coordination and consistency of each subsystem with the total goal.

According to the view of system theory, the meaning of the coordination degree is to reflect the coordination and consistency of each subsystem with the total goal. In this study, the coordination degree mainly refers to the balance of development of risk subsystem in each region. The smaller the differences of evaluation values are, the higher the coordination degree is. So, in the study a smaller coordination degree will show a more balanced situation in the region. The coordination degree can be calculated as the following formula:

$$
\mathrm{a}_{\mathrm{i}}=\frac{\mathrm{S}_{\mathrm{i}}}{\mathrm{M}_{\mathrm{i}}}
$$

where $S_{i}$ represents the mean of evaluating values of all subsystems in city $\mathrm{i}$, and $\mathrm{M}_{\mathrm{i}}$ represents the standard deviation. The greater the value of $a_{i}$ is, the lower the coordination degree of each subsystem is. Through calculation, we get the coordination degree of each region as shown in table III.

TABLE III. REGIONAL COORDINATION DEGREE

\begin{tabular}{cc||cc}
\hline region & $\begin{array}{c}\text { coordination } \\
\text { degree }\end{array}$ & region & $\begin{array}{c}\text { coordination } \\
\text { degree }\end{array}$ \\
\hline Xinjiang & 2.126 & Shanxi & 2.026 \\
Hainan & 2.124 & Hunan & 2.025 \\
Guizhou & 2.119 & Hubei & 1.995 \\
Xizang & 2.111 & Qinghai & 1.993 \\
Jilin & 2.098 & Liaoning & 1.987 \\
Guangxi & 2.097 & Henan & 1.987 \\
Jiangxi & 2.088 & Gansu & 1.981 \\
Shanxi & 2.085 & Zhejiang & 1.933 \\
Anhui & 2.081 & Shandong & 1.900 \\
Heilongjiang & 2.079 & Jiangsu & 1.834 \\
Chongqing & 2.077 & Shanghai & 1.806 \\
Ningxia & 2.039 & Guangdong & 1.769 \\
Fujian & 2.034 & Sichuan & 1.746 \\
Hebei & 2.033 & Tianjin & 1.704 \\
Yunnan & 2.028 & Beijing & 1.570 \\
Neimenggu & 2.028 & & \\
\hline
\end{tabular}

\section{CONCLUSION}

From five subsystems including supply risk, technical risk, market risk, natural risk and policy risk, ten indexes including material supply, stability of material supply, growth rate of power consumption, technology investment funds, accepted degree of people, natural disasters loss and so on, are selected to evaluate the risk of biomass power generation of subsystem, total system and coordination degree in 31 provinces or cities in China. What is more, we have analyzed the evaluation results, and according to the evaluation results a development direction and some relevant policy suggestions of biomass power generation are put forward.

Because there are so many indexes in the evaluation system, it is difficult to avoid the repeated information among the indexes and this may affect the last results in some certain.

To solve this problem, we can use some methods such as factor analysis or principal component analysis before we calculation and this may be a good idea. 


\section{REFERENCES}

[1] Junchen Wang, Lin Dai and Yishui Tian, "Analysis of the development status and trends of biomass energy industry in China, ”Transactions of the CSAE, vol. 23, pp. 276-282, September, 2007.

[2] Deyuan Wang, "Study on the comprehensive evaluation about the biomass energy systems, "Wuhan:Huazhong University of Science\&Technology, 2008, pp. 36-38.

[3] Jingchun Fan, Yonggang Wang and Shiping Qin, "Electricity price sensitive factor of biomass generation power, "Renewable Energy, vol. 126, pp. 49-51, February, 2006.

[4] Gang Hou, "research on the risk evaluation and management of biomass energy in China, ”Yangling:NorthwestA\&F University, 2009, pp. 67-78. 